\title{
Effect of Narrow Band Signal Interference Caused by CFO on OFDM System for Proximity-based Direct Communication
}

\author{
Seokki Kim*, Sungcheol Chang* \\ * Electronics and Telecommunications Research Institute (ETRI), Korea \\ kimsk0729@etri.re.kr, scchang@etri.re.kr
}

\begin{abstract}
Proximity-based direct communication is the technology that devices in the near distance can use the radio resource efficiently by operating distributedly without control of BS or AP. Many technologies are proposed for proximity-based direct communication and taking the operation in unlicensed band into account. Thus, coexistence techniques are needed for coexistence with different kind of devices which operate in unlicensed band such as Wireless LAN. A blocking signal transmission scheme to avoid contention with other devices by transmitting narrow band signal is proposed as one of the coexistence schemes for coexistence with different kind of devices. In this work, we present analysis of the effect of narrow band interference such as blocking signal caused by CFO on OFDM system for proximity-based direct communication and validate the theoretical results by means of computer simulation.
\end{abstract}

\section{Keywords — Direct Communication, Proximity, ICI, CFO}

\section{INTRODUCTION}

Recently, as wireless data traffic is rapidly increasing due to the spread of smart mobile devices, a need of new wireless communication system is coming to the fore to reduce the load of the wireless communication network and use the radio resource efficiently. Proximity-based direct communication is the technology that devices in the near distance can use the radio resource efficiently by operating distributedly without control of BS(Base Station) or AP(Access Point).

In that connection, standardization activities related to the proximity-based direct communication are proceeding briskly in many organizations. In IEEE 802.15.8 PAC(Peer Aware Communications) task group, especially, under the IEEE 802.15 WPAN(Wireless Personal Area Networks) work group, a project is underway to support proximity-based direct communication[1].

The PAC task group is taking the OFDM(Orthogonal Frequency Division Multiplexing) transmission technique[2] and operation in unlicensed band into account. Various element technologies are proposed in the PAC task group to meet the PAC requirements[3-4]. Among the rest, the distributed synchronization[5]-based contention-free multiple access scheme which is one of the proposals has several advantages such as scalability of the resource allocation to support a large number of devices, power saving and low overhead. However, coexistence techniques are needed for coexistence with different kind of devices which adopt the contention-based multiple access scheme such as Wireless LAN(Local Area Network) because the PAC is taking the operation in unlicensed band into account[6-7].

A blocking signal transmission scheme is proposed as one of the coexistence schemes for coexistence with different kind of devices. PAC devices which operate distributedly can avoid contention with different kind of devices by transmitting the blocking signal within certain interval and occupying the radio resource prior to other devices before their own data signal transmission. Only a few subcarriers are assigned for blocking signal transmission apart from data subcarriers. The blocking signal doesn't affect the data signal if we assume perfect frequency synchronization among PAC devices because there is no $\mathrm{ICI}($ Inter-Carrier Interference) caused by $\mathrm{CFO}$ (Carrier Frequency Offset). However, ICI occurs inescapably because the CFO exists among PAC devices due to the distributed operation of PAC devices. Also, large ICI can occur in spite of small CFO because the transmit power of blocking signal is concentrated in narrow band and data signal power to blocking signal power ratio may be very small according to the position of PAC devices. Therefore, performance degradation by blocking signal interference is needed to be evaluated for the system design.

In this work, we present analysis of the effect of the narrow band interference caused by CFO on OFDM system for proximity-based direct communication and validate the theoretical results by means of computer simulation.

\section{SYSTEM MODEL}

\section{A. OFDM Baseband Model with CFO}

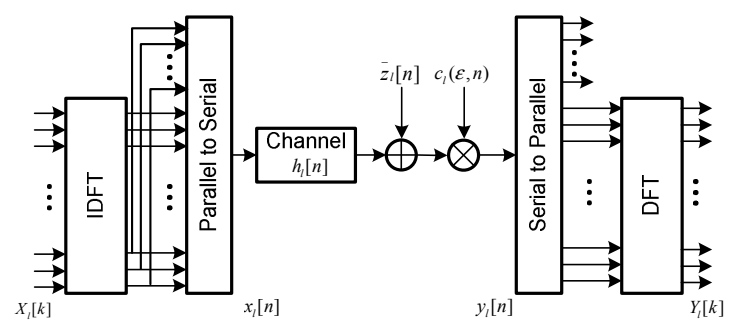

Figure 1. Discrete time baseband model of the OFDM system with CFO 
Figure 1 represents the baseband model of the OFDM system with CFO between transmitter and receiver. The transmitted frequency domain symbol $X_{l}[k]$ of $k$-th subcarrier with zero mean and variance $\sigma_{X}^{2}$ is transformed to the time domain sample by IDFT(Inverse Discrete Fourier Transform) and added $\mathrm{CP}($ Cyclic Prefix). The $n$-th time domain sample $x_{l}[n]$ at $l$-th symbol time is expressed as follows

$$
x_{l}[n]=\left\{\begin{array}{lc}
\frac{1}{\sqrt{N}} \sum_{k=0}^{N-1+N_{c p}} X_{l}[k] e^{j 2 \pi k\left(n-N_{c p}\right) / N}, \\
0, \quad \text { for } 0 \leq n \leq N+N_{c p} \\
\text { otherwise }
\end{array}\right.
$$

where $N$ and $N_{C P}$ are number of subcarriers and CP length, respectively.

The time domain sample $x_{l}[n]$ passes through the channel $h_{l}[n]$ and additive complex white Gaussian noise $\bar{z}_{l}[n]$ with zero mean and variance $\sigma_{z}^{2}$ is added. If the channel $h_{l}[n]$ is time-invariant within each OFDM symbol interval the time domain sample $\bar{y}_{l}[n]$ after channel and noise is expressed as follows

$$
\bar{y}_{l}[n]=h_{l}[n] * x_{l}[n]+\bar{z}_{l}[n]
$$

where $*$ is the convolution operator defined as

$$
h_{l}[n] * x_{l}[n] \stackrel{\Delta}{=} \sum_{t=-\infty}^{+\infty} h_{l}[t] x_{l}[n-t]
$$

And a phase rotation in proportion to $\mathrm{CFO} f_{o}$ between transmitter and receiver is occurred by frequency synchronization error. The phase rotation $c_{l}(\varepsilon, n)$ of $n$-th time domain sample $\bar{y}_{l}[n]$ at $l$-th symbol time can be written as follows

$$
c_{l}(\varepsilon, n)=e^{j 2 \pi \varepsilon\left(l\left(N+N_{c p}\right)+n-N_{c p}\right) / N}
$$

where $\varepsilon$ is the normalized CFO defined as

$$
\varepsilon \stackrel{\Delta}{=} \frac{f_{o}}{f_{\text {sub }}}
$$

where $f_{\text {sub }}$ is the subcarrier spacing.

So, we can express the received time domain sample $y_{l}[n]$ at the receiver side as follows

$$
\begin{aligned}
& y_{l}[n]=c_{l}(\varepsilon, n) \bar{y}_{l}[n] \\
& =c_{l}(\varepsilon, n)\left(h_{l}[n] * x_{l}[n]\right)+z_{l}[n]
\end{aligned}
$$

where $z_{l}[n]=c_{l}(\varepsilon, n) \bar{z}_{l}[n] \cdot z_{l}[n]$ is also a complex Gaussian random variable with zero mean and variance $\sigma_{z}^{2}$ because it is just phase rotation of the $\bar{z}_{l}[n]$. Also, we can define the SNR(Signal to Noise power Ratio) as follows

$$
S N R \stackrel{\Delta}{=} \frac{\sigma_{x}^{2}}{\sigma_{z}^{2}}
$$

The received frequency domain symbol $Y_{l}[k]$ is obtained by DFT(Discrete Fourier Transform) of the time domain sample $y_{l}[n]$ at $l$-th symbol time. The frequency domain symbol $Y_{l}[k]$ of $k$-th subcarrier at $l$-th symbol time is expressed as follows

$$
Y_{l}[k]=\frac{1}{\sqrt{N}} \sum_{n=N_{c p}}^{N-1+N_{c p}} y_{l}[n] e^{j 2 \pi k\left(n-N_{c p}\right) / N}
$$

If we assume that the delay spread of the channel $h_{l}[n]$ is smaller than CP length, there is no ISI(Inter-Symbol Interference). Therefore, $Y_{l}[k]$ is expressed as follows

$$
Y_{l}[k]=C_{l}(\varepsilon, k) *\left(H_{l}[k] X_{l}[k]\right)+Z_{l}[k]
$$

where $C_{l}(\varepsilon, k), H_{l}[k], Z_{l}[k]$ is the DFT output of $c_{l}(\varepsilon, n)$, $h_{l}[n]$ and $z_{l}[n]$, respectively.

If the normalized CFO is $-0.5 \leq \varepsilon \leq 0.5, C_{l}(\varepsilon, k)$ can be written as

$$
C_{l}(\varepsilon, k)=\sum_{n=N_{c p}}^{N-1+N_{c p}} c_{l}(\varepsilon, n) e^{-j 2 \pi\left(n-N_{c p}\right) k / N}
$$

And if we assume that $H_{l}[k]$ and $X_{l}[k]$ periodic with period $N$ in frequency domain after DFT, the received frequency domain symbol $Y_{l}[k]$ of (9) can be expressed as follows

$$
\begin{aligned}
& Y_{l}[k]=\sum_{r=0}^{N-1} C_{l}(\varepsilon, r) H_{l}[k-r] X_{l}[k-r]+Z_{l}[k] \\
& =C_{l}(\varepsilon, 0) H_{l}[k] X_{l}[k] \\
& +\sum_{r=1}^{N-1} C_{l}(\varepsilon, r) H_{l}[k-r] X_{l}[k-r]+Z_{l}[k]
\end{aligned}
$$

And $C_{l}(\varepsilon, r)$ of $(10)$ which is the DFT output of $c_{l}(\varepsilon, n)$ can be expressed as follows[8-9]

$$
C_{l}(\varepsilon, k)=\left(\frac{\sin (\pi(\varepsilon-k))}{N \sin (\pi(\varepsilon-k) / N)} e^{j \pi(\varepsilon-k)(1-1 / N)}\right) e^{j\left(2 \pi \varepsilon l\left(1+N_{c p} / N\right)\right)}
$$

\section{B. Blocking Signal Transmission Scheme}

As mentioned above, PAC is taking the operation in unlicensed band into account. Thus, the contention may occur between PAC devices and different kind of devices which adopt the contention-based multiple access scheme due to the feature of the PAC devices which operate distributedly. Therefore, the PAC devices which operate on synchronous mode based on distributed synchronization scheme require coexistence techniques for coexistence with other devices such as Wireless LAN which operate in unlicensed band.

The PAC devices can avoid the contention with other devices by transmitting blocking signal within certain interval and occupying the radio resource prior to other devices before their own data signal transmission as represented by figure 2 .

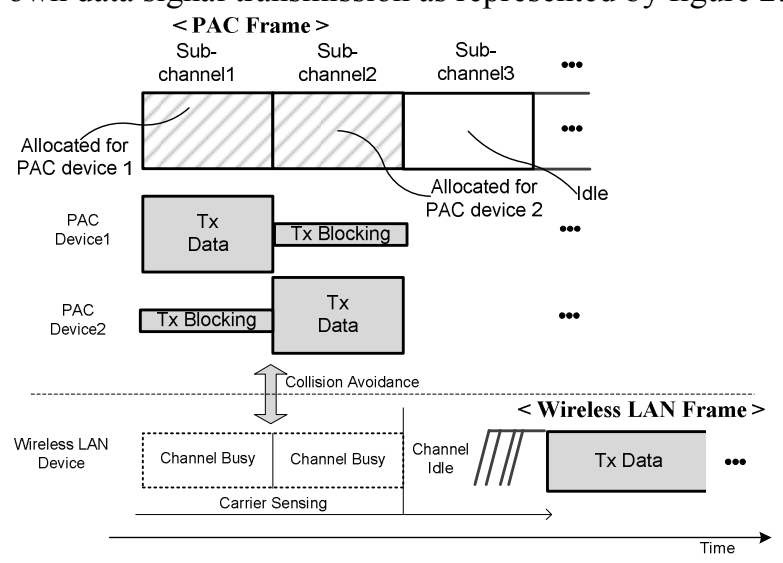

Figure 2. Coexistence scheme by blocking signal transmission 
The subcarriers for data and blocking signal transmission can be simply allocated as figure 3 although there are many allocation schemes.

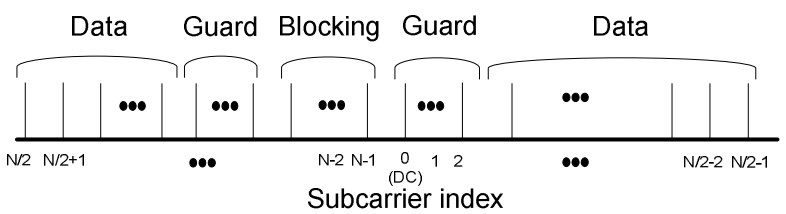

Figure 3. Subcarrier frequency allcoation for data and blocking symbol

\section{Interference Scenario}

Only a few subcarriers are assigned for blocking signal transmission separated from data subcarriers. The blocking signal doesn't affect the data signal if we assume perfect frequency synchronization because there is no ICI caused by CFO. However, the ICI exists necessarily because the CFO exists among PAC devices due to the fully distributed operation of PAC devices.

The Rx PAC device estimates and compensates the CFO $\varepsilon_{1}$ of the Tx PAC device to decode data signal. But the CFO $\varepsilon_{2}$ of a PAC device which transmits the blocking signal is not considered. Thus, there exist CFO $\Delta \varepsilon=\varepsilon_{2}-\hat{\varepsilon}_{1}$ between the $\mathrm{Rx}$ PAC device and a PAC device which transmits the blocking signal. $\hat{\varepsilon}_{1}$ is the estimation of $\varepsilon_{1}$. Also, large ICI can occur in spite of small $\Delta \varepsilon$ because data signal to blocking signal power ratio may be very small according to the position of PAC devices as we can see in the figure 4.

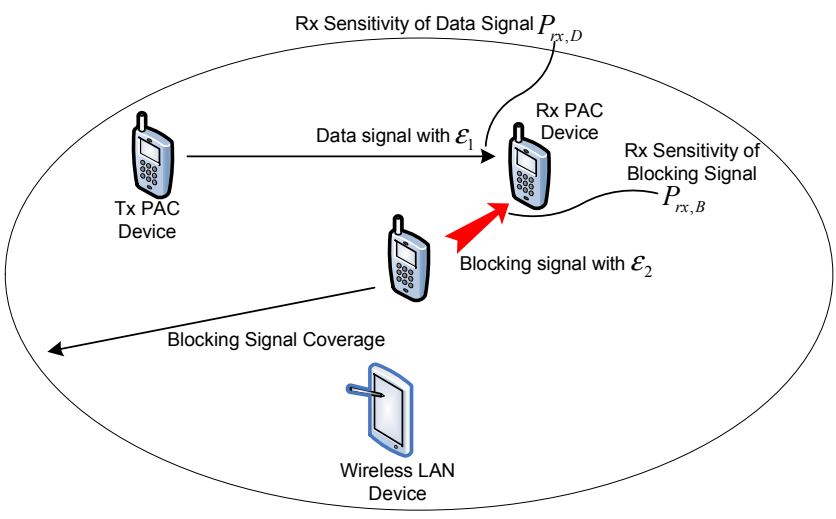

Figure 4. Interference by blocking signal

We can define SIR(signal to interference power ratio) as follows

$$
\operatorname{SIR} \stackrel{\Delta}{=} \frac{P_{r x, D}}{P_{r x, B}}
$$

where $P_{r x, D}$ and $P_{r x, B}$ are the receive sensitivity of the data signal and blocking signal, respectively. Furthermore, the transmit power of blocking signal is concentrated in narrow band(e.g. only a few subcarriers for blocking signal transmission) to maintain that the coverage of the blocking signal is equal to the coverage of the data signal. In this case, the transmit power of blocking symbol is defined as

$$
\sigma_{B}^{2} \stackrel{\Delta}{=} \frac{N_{D}}{N_{B}} \sigma_{X}^{2}
$$

where $N_{D}$ and $N_{B}$ are the number of subcarriers for data symbol transmission and the number of subcarriers for blocking symbol transmission, respectively.

\section{III.SINR ANALYSIS}

From the interference scenario of the figure 4, the received time domain sample $y_{l}[n]$ of the receiver is expressed as follows

$$
y_{l}[n]=y_{l, D}[n]+\sqrt{\frac{1}{S I R}} y_{l, B}[n]+z_{l}[n]
$$

The $y_{l, D}[n]$ and $y_{l, B}[n]$ which are received time domain sample from desired signal transmitter and blocking signal transmitter are expressed as follows

$$
y_{l, D}[n]=\left\{\begin{array}{cc}
c_{l}\left(\varepsilon_{1}-\hat{\varepsilon}_{1}, n\right) h_{l, D}[n] * \frac{1}{\sqrt{N}} \sum_{k=0}^{N-1+N_{c p}} D_{l}[k] e^{j 2 \pi k\left(n-N_{c p}\right) / N}, \\
\text { for } 0 \leq n \leq N+N_{c p} \\
0, \quad \text { otherwise }
\end{array}\right.
$$

and

$$
y_{l, B}[n]=\left\{\begin{array}{cc}
c_{l}(\Delta \varepsilon, n) h_{l, B}[n] * \frac{1}{\sqrt{N}} \sum_{k=0}^{N-1+N_{c p}} B_{l}[k] e^{j 2 \pi k\left(n-N_{c p}\right) / N}, \\
\text { for } 0 \leq n \leq N+N_{c p} \\
0, \quad \text { otherwise }
\end{array}\right.
$$

where $h_{l, D}[n]$ is the channel between desired signal transmitter and receiver, and $h_{l, B}[n]$ is the channel between blocking signal transmitter and receiver. And $D_{l}[k]$ and $B_{l}[k]$ are as follows

$$
D_{l}[k]= \begin{cases}d_{l, k}, & k \in \mathrm{K}_{D} \\ 0 & , k \notin \mathrm{K}_{D}\end{cases}
$$

and

$$
B_{l}[k]= \begin{cases}b_{l, k} & , k \in \mathrm{K}_{\mathrm{B}} \\ 0 & , k \notin \mathrm{K}_{\mathrm{B}}\end{cases}
$$

where $d_{l, k}$ and $\mathbf{K}_{\mathbf{D}}$ are the transmitted data symbol stream and the set of the subcarrier index for data symbol, respectively. And $b_{l, k}$ and $\mathbf{K}_{\mathbf{B}}$ are the blocking symbol stream and the set of the subcarrier index for blocking symbol, respectively. From the definition of (14), the variance of the $b_{l, k}$ is $\sigma_{B}^{2}=N_{D} / N_{B} \cdot \sigma_{X}^{2}$

The received frequency domain symbol $Y_{l}[k]$ which is DFT output of $y_{l}[n]$ is expressed as follows

$$
Y_{l}[k]=Y_{l, D}[k]+\sqrt{\frac{1}{S I R}} Y_{l, B}[k]+Z_{l}[k]
$$

where $Y_{l, D}[k]$ and $Y_{l, B}[k]$ are the DFT output of the $y_{l, D}[n]$ and $y_{l, B}[n]$, respectively. From (11), $Y_{l, D}[k]$ and $Y_{l, B}[k]$ can be written as

$$
Y_{l, D}[k]=C_{l}\left(\varepsilon_{1}-\hat{\varepsilon}_{1}, 0\right) H_{l, D}[k] D_{l}[k]+I C I_{\text {data }}
$$




$$
Y_{l, B}[k]=C_{l}(\Delta \varepsilon, 0) H_{l, B}[k] B_{l}[k]+I C I_{\text {blocking }}
$$

where the $H_{l, D}[k]$ and $H_{l, B}[k]$ is the DFT output of the $h_{l, D}[n]$ and $h_{l, B}[n]$, respectively. The $I C I_{\text {data }}$ and $I C I_{\text {blocking }}$ are the interferences which are caused by $\varepsilon_{1}-\hat{\varepsilon}_{1}$ and $\Delta \varepsilon$. These interferences are expressed as follows

$$
I C I_{d a t a}=\sum_{r=1}^{N-1} C_{l}\left(\varepsilon_{1}-\hat{\varepsilon}_{1}, r\right) H_{l, D}[k-r] D_{l}[k-r]
$$

and

$$
I C I_{\text {blocking }}=\sum_{r=1}^{N-1} C_{l}(\Delta \mathcal{E}, r) H_{l, B}[k-r] B_{l}[k-r]
$$

Because the subcarriers for blocking symbol transmission are separately allocated from the subcarriers for data symbol transmission, $C_{l}(\Delta \varepsilon, 0) H_{l, B}[k] B_{l}[k]$ of (22) doesn't affect the desired signal. Thus, for given channel $H_{l, D}[k], H_{l, B}[k]$, normalized frequency offset $\varepsilon_{1}, \varepsilon_{2}$ and SIR, the signal-tonoise and interference power ratio SINR of any subcarrier index at any symbol time is obtained as follows

$$
\operatorname{SINR}=\frac{E\left[\left|C_{l}\left(\varepsilon_{1}-\hat{\varepsilon}_{1}, 0\right) H_{l, D}[k] X_{l}[k]\right|^{2}\right]}{E\left[\left|I C I_{\text {data }}+\sqrt{\frac{1}{S I R}} I C I_{\text {blocking }}+Z_{l}[k]\right|^{2}\right]}
$$

Assuming $\varepsilon_{1}$ is perfectly estimated and compensated by the receiver, $C_{l}\left(\varepsilon_{1}-\hat{\varepsilon}_{1}, 0\right)$ is 1 and $I C I_{\text {data }}$ is 0 . Thus there is no impact of the interference by $\varepsilon_{1}-\hat{\varepsilon}_{1}$. Also, if we assume that the $H_{l, D}[k]$ is circular symmetry complex Gaussian random variable with zero mean and variance $\sigma_{H}^{2}$, the power of desired signal is equal to

$$
\begin{aligned}
& E\left[\left|C_{l}\left(\varepsilon_{1}-\hat{\varepsilon}_{1}, 0\right) H_{l, D}[k] X_{l}[k]\right|^{2}\right] \\
& =E\left[\left|H_{l, D}[k]\right|^{2}\right] E\left[\left|X_{l}[k]\right|^{2}\right]=\sigma_{H}^{2} \sigma_{X}^{2}
\end{aligned}
$$

because $H_{l, D}[k]$ is independent of $X_{l}[k]$.

$I C I_{\text {data }}, I C I_{\text {blocking }}$ and $Z_{l}[k]$ are also independent each other because $X_{l}[k], B_{l}[k]$ and $Z_{l}[k]$ are independent each other and $I C I_{\text {data }}$ and $I C I_{\text {blocking }}$ are the linear combination of $X_{l}[k]$ and $B_{l}[k]$, respectively. So the power of noise and interference is equal to

$$
\begin{aligned}
& E\left[\left|I C I_{\text {data }}+\sqrt{\frac{1}{S I R}} I C I_{\text {blocking }}+Z_{l}[k]\right|^{2}\right] \\
& =E\left[\left|I C I_{\text {data }}\right|^{2}\right]+\frac{1}{S I R} E\left[\left|I C I_{\text {blocking }}\right|^{2}\right]+E\left[\left|Z_{l}[k]\right|^{2}\right]
\end{aligned}
$$

In (22), the blocking symbol $B_{l}[k]$ which passes through the channel $H_{l, B}[k]$ is distorted by $C_{l}(\Delta \varepsilon, 0)$. Also the interference $C_{l}(\Delta \varepsilon, r) H_{l, B}[k-r] B_{l}[k-r]$ generated by $B_{l}[k-r]$, which is located $r$ subcarriers apart from $B_{l}[k]$, is added to the $C_{l}(\Delta \varepsilon, 0) H_{l, B}[k] B_{l}[k]$. Considering the interference from a different point of view, we can define a interference generated by $B_{l}[k]$, which is located $r$ subcarriers apart from $B_{l}[k]$, as follows

$$
i(\Delta \varepsilon, r, k) \stackrel{\Delta}{=} C_{l}(\Delta \varepsilon, r) H_{l, B}[k] B_{l}[k]
$$

Thus, the summation of the interference generated by $B_{l}[k]$ at any symbol time can be expressed as follows

$$
i_{\text {sum }}=\sum_{k=0}^{N-1}\left(\sum_{r=1}^{N-1} i(\Delta \varepsilon, r, k)\right)
$$

But the $i_{\text {sum }}$ is not equal to the interference which affects the desired signal. $i(\Delta \varepsilon, r, k)$ affects the desired signal only if a subcarrier, which is located $r$ subcarriers apart from $B_{l}[k]$, is allocated for data symbol. So we can express the average interference power $P_{I}(\Delta \varepsilon)$ which affects each data subcarrier at any symbol time as follows

$$
P_{I}(\Delta \varepsilon)=\frac{1}{N_{D}} \sum_{\substack{k=0 \\ k \in \mathbf{K}_{\mathbf{B}}}}^{N-1}\left(\sum_{\substack{r=1 \\ \bmod (k+r, N) \in \mathbf{K}_{\mathbf{D}}}}^{N-1}|i(\Delta \varepsilon, r, k)|^{2}\right)
$$

Therefore the power of interference by blocking signal is calculated as

$$
\begin{aligned}
& E\left[\left|I C I_{\text {blocking }}\right|^{2}\right]=E\left[P_{I}(\Delta \varepsilon)\right] \\
& =\frac{1}{N_{D}} \sum_{\substack{k=0 \\
k \in \mathbf{K}_{\mathbf{B}}}}^{N-1}\left(\sum_{\substack{r=1 \\
\bmod (k+r, N) \in \mathbf{K}_{\mathbf{D}}}}^{N-1} E\left[|i(\Delta \varepsilon, r, k)|^{2}\right]\right)
\end{aligned}
$$

If we assume that the $H_{l, B}[k]$ is also circular symmetry complex Gaussian random variable with zero mean and variance $\sigma_{H}^{2}$

$$
\begin{aligned}
E\left[|i(\Delta \mathcal{\varepsilon}, r, k)|^{2}\right] & =E\left[\left|C_{l}(\Delta \mathcal{\varepsilon}, r) H_{l, B}[k] B_{l}[k]\right|^{2}\right] \\
& =\left|C_{l}(\Delta \mathcal{\varepsilon}, r)\right|^{2} \sigma_{H}^{2} \sigma_{B}^{2}
\end{aligned}
$$

because $H_{l, B}[k]$ is independent of $B_{l}[k]$. Therefore, if the data and blocking symbol subcarriers are allocated as figure 3 , according to the $N_{B}$, (31) can be written as

$$
\begin{aligned}
& E\left[\mid \text { ICI }\left._{\text {blocking }}\right|^{2}\right] \\
& =\left\{\begin{array}{lr}
\frac{\sigma_{H}^{2} \sigma_{B}^{2}}{N_{D}} \sum_{r=1}^{N-1}\left|C_{l}(\Delta \varepsilon, r)\right|^{2}, & \text { if } N_{B}=1 \\
\frac{\sigma_{H}^{2} \sigma_{B}^{2}}{N_{D}}\left(\sum_{r=2}^{N-1}\left|C_{l}(\Delta \varepsilon, r)\right|^{2}+\sum_{r=1}^{N-2}\left|C_{l}(\Delta \varepsilon, r)\right|^{2}\right), & \\
\frac{\sigma_{H}^{2} \sigma_{B}^{2}}{N_{D}}\left(\sum_{r=3}^{N-1}\left|C_{l}(\Delta \varepsilon, r)\right|^{2}+\sum_{r=2}^{N-2}\left|C_{l}(\Delta \varepsilon, r)\right|^{2}+\sum_{r=1}^{N-3}\left|C_{l}(\Delta \varepsilon, r)\right|^{2}\right) \\
\vdots
\end{array}\right. \\
& \text { if } N_{B}=3
\end{aligned}
$$

And (33) can be expressed as follows

$$
\begin{aligned}
& E\left[\left|I C I_{\text {blocking }}\right|^{2}\right] \\
& =\frac{\sigma_{H}^{2} \sigma_{B}^{2}}{N_{D}}\left(N_{B} \sum_{r=N_{B}}^{N-N_{B}}\left|C_{l}(\Delta \varepsilon, r)\right|^{2}-\sum_{r=1}^{N_{B}-1}\left|C_{l}(\Delta \varepsilon, r)\right|^{2}-\sum_{r=N-N_{B}+1}^{N-1}\left|C_{l}(\Delta \varepsilon, r)\right|^{2}\right)
\end{aligned}
$$

From (34), we can find that the power of interference by blocking signal is not related to the subcarrier index $k$ for 
blocking symbol. Also, from (12), following relationship is established.

$$
\sum_{r=u}^{N-v}\left|C_{l}(\Delta \varepsilon, r)\right|^{2}=\sum_{r=v}^{N-u}\left|C_{l}(\Delta \varepsilon, r)\right|^{2}
$$

Thus, (34) can be modified as

$$
E\left[\left|I C I_{\text {blocking }}\right|^{2}\right]=\frac{\sigma_{H}^{2} \sigma_{B}^{2}}{N_{D}}\left(N_{B} \sum_{r=N_{B}}^{N-N_{B}}\left|C_{l}(\Delta \varepsilon, r)\right|^{2}-2 \sum_{r=1}^{N_{B}-1}\left|C_{l}(\Delta \varepsilon, r)\right|^{2}\right)
$$

Substituting (14) for $\sigma_{B}^{2},(36)$ is finally expressed as follows

$$
\begin{aligned}
& E\left[\left|I C I_{\text {blocking }}\right|^{2}\right] \\
& =\sigma_{H}^{2} \sigma_{X}^{2}\left(\sum_{r=N_{B}}^{N-N_{B}}\left|C_{l}(\Delta \varepsilon, r)\right|^{2}-\frac{2}{N_{B}} \sum_{r=1}^{N_{B}-1}\left|C_{l}(\Delta \varepsilon, r)\right|^{2}\right)
\end{aligned}
$$

If some guard subcarriers are allocated to reduce the interference by blocking signal and we assume that it's even number, (37) can be simply modified as

$$
\begin{aligned}
& E\left[\left|I C I_{\text {blocking }}\right|^{2}\right] \\
& =\sigma_{H}^{2} \sigma_{X}^{2}\left(\sum_{r=N_{B}+N_{G} / 2}^{N-N_{B}-N_{G} / 2}\left|C_{l}(\Delta \varepsilon, r)\right|^{2}-\frac{2}{N_{B}} \sum_{r=1+N_{G} / 2}^{N_{B}-1+N_{G} / 2}\left|C_{l}(\Delta \varepsilon, r)\right|^{2}\right)
\end{aligned}
$$

where $N_{G}$ is the number of subcarriers for guard symbol. As we can see in (38), the interference by the blocking signal is expressed as a function of $N_{B}, N_{G}$ and $\Delta \varepsilon$.

At last, the power of $Z_{I}\left[k_{l, D}\right]$ is equal to

$$
E\left[\left|Z_{l}\left[k_{l, D}\right]\right|^{2}\right]=\sigma_{z}^{2}
$$

From (26), (38) and (39), we can calculate the SINR and SNR degradation $D\left(\Delta \varepsilon, N_{B}, N_{G}\right)$ which is defined as follows

$$
D\left(\Delta \varepsilon, N_{B}, N_{G}\right) \stackrel{\Delta}{=} \frac{S N R}{S I N R}
$$

\section{IV.SIMULATION}

In this section, we conduct computer simulation to verify the reliability of theoretical SNR degradation of (40). The SNR degradation is numerically calculated for various $S I R$, $N_{B}, N_{G}$, and $\Delta \varepsilon$. The SNR degradation is also obtained by computer simulation. The simulation is performed in AWGN channel. 64 subcarriers and $312.5 \mathrm{kHz}$ subcarrier spacing are chosen for OFDM transmission scheme.

Figure 5-7 show the SNR degradation according to the $\mathrm{CFO}$ and SIR. We assume that the numbers of blocking and guard subcarriers are 1 and 0 , respectively. The lines are theoretical results which are numerically calculated by (40) and markers are the simulation results.

If the SIR is large, SNR degradation by CFO is small enough to be ignored as figure 5. However, the larger CFO and the SIR are, the larger SNR degradations are. Furthermore, the SNR degradation increases even if the CFO is small when the SIR is small. Also, we can observe that the SNR degradation increase at high SNR because the impact of interference become increased relatively.

As mentioned in section $I$, large ICI can occur in spite of small $\Delta \varepsilon$ as figure 7 due to the fully distributed operation of proximity-based direct communication system. To reduce
SNR degradation, we can increase the number of subcarriers for blocking and guard symbols. Figure 8 and 9 show the SNR degradation according to the number of subcarriers for blocking and guard symbols in case of low SIR(-20dB). These results can provide the design criteria to meet the requirement of the target system.

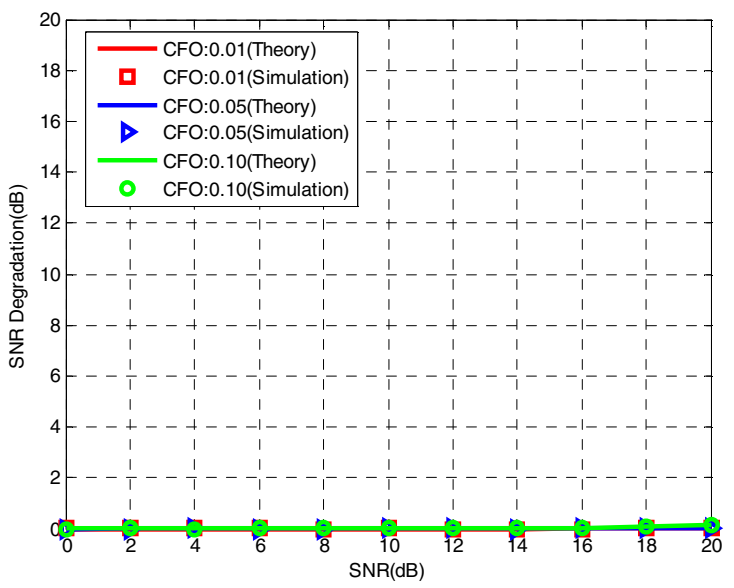

Figure 5. SINR degradation in case of SIR:20dB

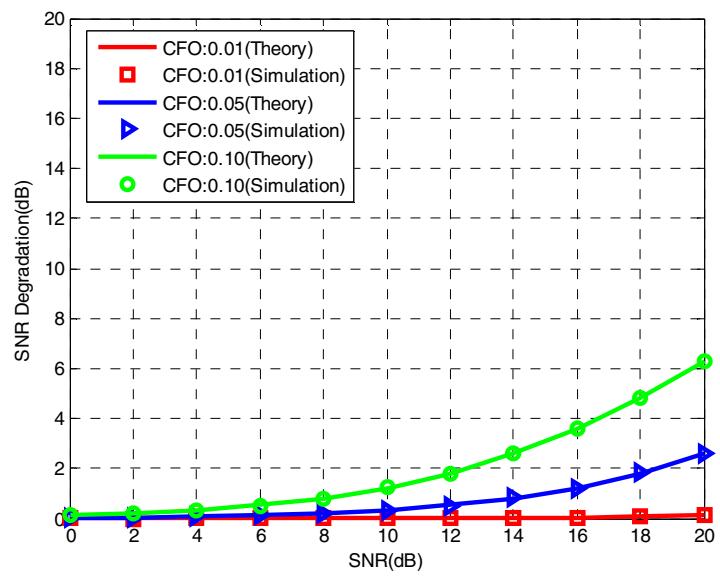

Figure 6. SINR degradation in case of SIR:0dB

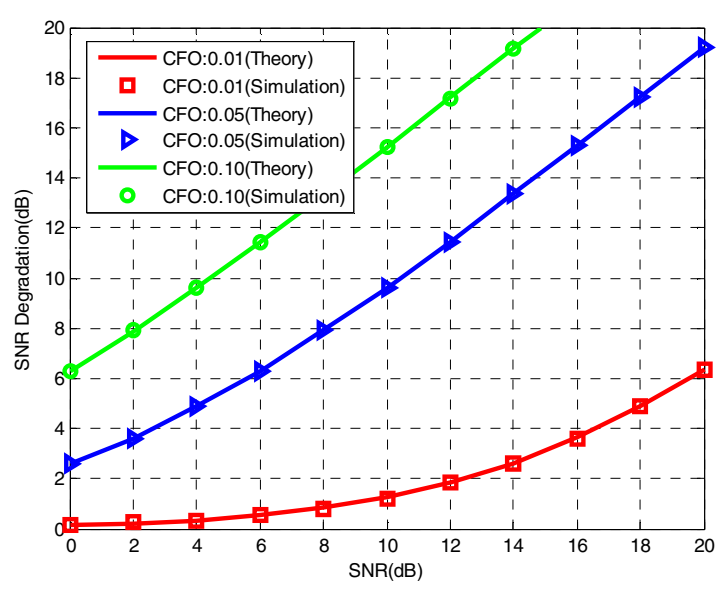

Figure 7. SINR degradation in case of SIR:-20dB 
As we can see in these figures, the theoretical results are very little different from the simulation results regardless of the various CFOs, SIRs and subcarrier allocations for blocking and guard symbols.

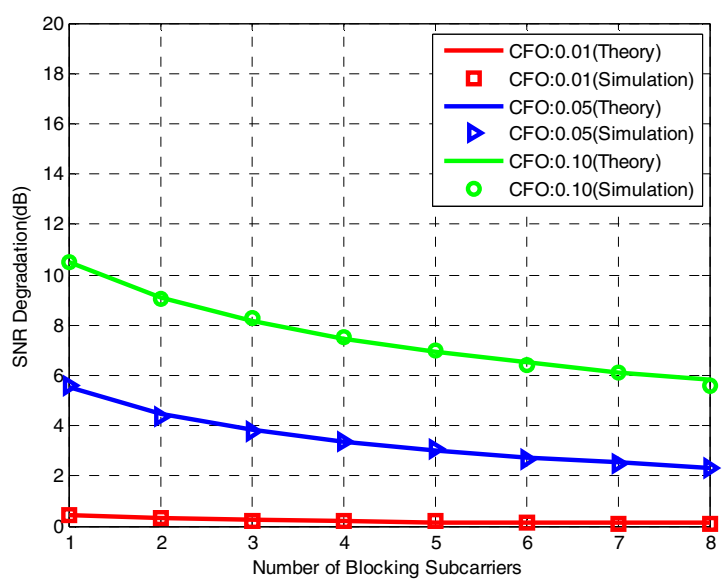

Figure 8. SINR degradation according to $N_{B}$ in case of SNR: $5 \mathrm{~dB}$

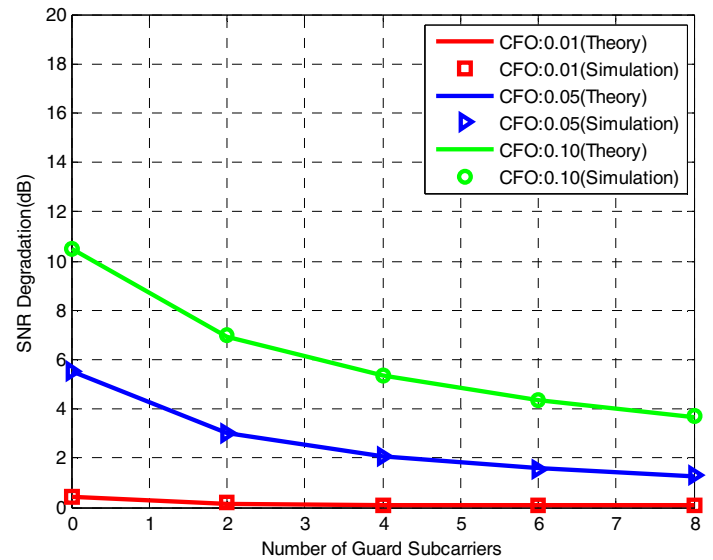

Figure 9. SINR degradation according to $N_{G}$ in case of SNR: $5 \mathrm{~dB}$

\section{Conclusions}

In this paper, we have presented the analysis of the effect of the narrow band interference such as blocking signal of PAC caused by CFO on OFDM system for proximity-based direct communication. The SNR degradation can be expressed as a function of CFO, number of subcarriers for blocking and guard symbols. The theoretical result was validated by means of computer simulation. The impact of the interference could be handled by adjustment of subcarrier allocation for blocking and guard symbols. The analytic expression can be helpful to design the OFDM system for proximity-based direct.

\section{ACKNOWLEDGMENT}

This research was funded by the MSIP(Ministry of Science, ICT \& Future Planning), Korea in the ICT R\&D Program 2013.

\section{REFERENCES}

[1] The IEEE 802.15.8 PAC website. Available: http://www.ieee802.org/15/pub/TG8.html.

[2] J. A. C. Bingham, "Multicarrier modulation for data transmission: An idea whose time has come," IEEE Comm. Mag., vol. 28, pp. 5-14, May 1990

[3] TG8 Technical Guidance Document, IEEE 802.15.12-0568-07-0008, Nov. 1, 2012.

[4] TG8 PAC Framework Document (Draft), IEEE 802.15.8-13-0328-080008, May 16, 2013.

[5] Osvaldo Simeone, "Distributed Synchronization in Wireless Networks," IEEE Signal Processing Magazine, Sep., 2008

[6] S. Mangold, J. Habetha, S. Choi, and C. Ngo, "Co-existence and Interworking of IEEE 802.11a and ETSI BRAN HiperLAN/2 in MultiHop Scenarios," in Proc. of IEEE 3rd Workshop in Wireless Local Area Networks, Boston, USA, 27-28 September 2001.

[7] Lars Berlemann, "Unlicensed Operation of IEEE 802.16: Coexistence with $802.11(\mathrm{~A})$ in Shared Frequency Bands," $17^{\text {th }}$ Annual IEEE international Symposium on PIMRC, 2006.

[8] W. Hwang, H. Kang, and K. Kim, "Approximation of SNR degradation due to carrier frequency offset for OFDM in shadowed multipath channels," IEEE Comm. Letters, vol. 7, pp. 581-583, Dec. 2003.

[9] Jungwon Lee, Hui-Ling Lou, Dimitris Toumpakaris and John M. Cioffi, "Effect of Carrier Frequency Offset on OFDM Systems for Multipath Fading Channel," IEEE Communication Society Globecom, 2004. 